\title{
Validación de un instrumento para medir competencias conductuales en personas VIH positivas
}

Julio Alfonso Piña-López, PMC.(1)

\begin{abstract}
Piña-López JA.
Validación de un instrumento para medir competencias conductuales en personas VIH positivas. Salud Publica Mex 2003;45:293-297.

El texto completo en inglés de este artículo está disponible en: http://www.insp.mx/salud/index.html
\end{abstract}

\section{Resumen}

Objetivo. Estudiar la confiabilidad y validez de un instrumento que mide competencias conductuales en personas que viven conVIH/sida. Material y métodos Estudio transversal, efectuado en los meses de noviembre y diciembre de 2002 en la ciudad de Hermosillo, estado de Sonora, México. En él se manejó un instrumento que consta de 24 reactivos y fue aplicado a una muestra de 60 personas VIH positivas que reciben atención en dos instituciones del sector salud, México. Se emplearon los siguientes procedimientos estadísticos: para la discriminación de los reactivos se utilizó la prueba t; para la validez de constructo se efectuó un análisis de su estructura factorial, mientras que para la confiabilidad se recurrió al estadístico alfa de Cronbach. Resultados Excepto dos reactivos, el resto discriminó correctamente; el análisis factorial arrojó tres factores, que en su conjunto explican $47.99 \%$ de la varianza, y un índice conformado por dos reactivos; finalmente, la encuesta en su conjunto alcanzó un alfa de Cronbach de 0.856 . Conclusiones. Este instrumento tiene las ventajas de ser claro, con una buena discriminación de los reactivos y de poseer validez de constructo. El texto completo en inglés de este artículo está disponible en: http://www.insp.mx/salud/ index.html

Palabras clave: confiabilidad; validez; competencias conductuales;VIH/SIDA; México

\author{
Piña-López JA. \\ Validation of an instrument to measure behavioral \\ competencies in HIV+ persons. \\ Salud Publica Mex 2003;45:293-297. \\ The English version of this paper \\ is available at: http://www.insp.mx/salud/index.html
}

\begin{abstract}
A bstract
Objective.To assess the reliability and validity of an instrument to measure behavio ral competences in HIV/AIDS-infected persons. Material and Methods.A cross-sectional study was conducted in N ovember and D ecember 2002 in Hermosillo, Sonora State, Mexico.An instrument consisting of 24 items was applied to 60 HIV-positive subjects who received health care through two Mexican Ministry of $\mathrm{H}$ ealth institutions. Statistical analysis consisted of Student s t test for item discrimination and Cronbach $\mathrm{s}$ alpha for testing construct reliability. Results All but two items were able to discriminate correctly; factorial analysis resulted in three factors that jointly accounted for $48 \%$ of the variance. Finally a two-item index was obtained. The survey as a whole attained a Cronbach $s$ alpha of 0.856 . Conclusions. And advantage of this instrument is that it is clear, as it has good item discrimination.In addition, this instrument has construct validity. The English version of this paper is available at: http://www.insp.mx/salud/index.html.
\end{abstract}

Key words:behavioral competencies; reliability; validity; HIV/ AID S; Mexico

\section{AIDS; Mexico}

1) Centro de Información para la Salud. Secretaría de Salud del Estado de Sonora. Hermosillo, Sonora, México.

Fecha de recibido: 3 de febrero de 2003 - Fecha de aprobado: 21 de julio de 2003

Solicitud de sobretiros: PMC. Julio Alfonso Piña López. Guillermo Prieto 18, Colonia Constitución 83150. Hermosillo, Sonora, México.

Correo electrónico: jpina@ salud.gob.mx 
$\mathrm{H}$ oy en día se dispone de una amplia variedad de instrumentos que tienen por objeto identificar qué variables psicológicas y/o sociales afectan de manera especial a las personas VIH positivas, dentro de los cuales destacan los que miden aspectos relacionados con la práctica de conductas de adhesión a la terapéutica médica, ${ }^{1}$ estados de depresión, ansiedad o estrés, ${ }^{2,3}$ funcionamiento social en general, ${ }^{4}$ sufrimiento percibido, ${ }^{5}$ locus de control, ${ }^{6}$ así como calidad de vida, ${ }^{7}$ entre otros. Sin embargo, poco se ha hecho por la construcción y validación de instrumentos que nos permitan medir aspectos relacionados con la variable personalidad y la noción de competencias conductuales, definida la primera como los modos consistentes de comportamiento (estilos interactivos) frente a una situación en la que no se prescribe ningún criterio de eficacia para el individuo, esto es, en una situación interactiva en la que no hay criterios explícitos sobre cómo un individuo debe comportarse ${ }^{8}$ y las segundas, como el conjunto de recursos que posee un individuo producto de su historia psicológica o interactiva, y que ha utilizado en el pasado para responder de manera efectiva en una situación. ${ }^{9}$

En el modelo psicológico de salud biológica, 10,11 las competencias conductuales, junto con las variables personalidad (estilos interactivos) y la modulación de los estados biológicos de respuesta, se inscriben dentro de la fase de procesos. Se trata de tres factores que son pertinentes para dar cuenta, desde un punto de vista psicológico, del mantenimiento, recuperación o pérdida de la salud. En el caso concreto de las competencias conductuales, para su análisis es necesario tener presentes tres aspectos, a saber: a) los requerimientos o demandas de una situación; b) los factores que participan en una situación, que comprenden el tipo de escenario, las condiciones físicas y sociales que la configuran, las personas que auspician la interacción, etcétera, y c) la historia de competencias, esto es, la capacidad que se tiene para responder de manera efectiva en una situación a partir de la experiencia. ${ }^{12}$

El propósito del presente estudio fue el de evaluar las propiedades psicométricas de un instrumento construido para medir competencias conductuales en personas VIH positivas. Se partió de la consideración teórica de que en las personas VIH positivas, el que se posea o no las competencias conductuales necesarias contribuirá, entre otras cosas, a que se cumpla o no con las indicaciones o sugerencias que brinda el personal de salud en su conjunto, o a que se practiquen o no conductas ajustivas de diferente tipo, tanto en lo que se refiere al propio cuidado de la salud, como al mantenimiento y mejoramiento de las relaciones sociales cotidianas.

\section{Material y métodos}

El presente estudio, de corte transversal, se realizó en el transcurso de los meses de noviembre y diciembre de 2002. Se contó con una muestra de 60 personas VIH positivas, quienes en esas fechas recibían atención en el Centro de Información para la Salud (CISAL), dependiente de la Secretaría de Salud del estado de Sonora y en el Hospital General Zona 2, "Adolfo Félix Lostaunau" dependiente del Instituto Mexicano del Seguro Social (IMSS). Los participantes fueron 35 del sexo masculino y 25 del sexo femenino, cuyas edades oscilaron entre los 19 y 55 años, con una media de 36.50 años de edad (DE=8.86). Al momento del estudio contaban con un promedio de escolaridad de 8.4 años por participante, equivalente a estudios entre el segundo y tercer año de secundaria; adicionalmente, $78 \%$ de los participantes reportaron estar solteras o solteros y el restante $22 \%$ vivía en pareja, eran divorciadas o divorciados o se encontraban separadas o separados. Finalmente, de acuerdo con su historial médico el tiempo de infección por el VIH fluctuó en un rango de 6 a 143 meses, con una media de 54.03 meses (DE=32.74).

La medición de las competencias conductuales se llevó a cabo con un instrumento construido ex profeso, el cual consta de 24 reactivos. Las respuestas a los reactivos se evaluaron en un formato tipo Likert que incluía cuatro o cinco opciones. Un ejemplo de reactivo con cinco opciones era el siguiente: "Poseo información completa sobre los posibles efectos secundarios que pueden llegar a producir los medicamentos antiretrovirales", con respuestas que iban de $1=$ totalmente de acuerdo a $5=$ totalmente en desacuerdo; un reactivo con cuatro opciones de respuesta era: "Consumo los medicamentos según se necesite, unos acompañados de líquidos y otros con alimentos", donde 1= siempre, $2=$ casi siempre, $3=$ a veces y $4=$ nunca. Por último, es conveniente mencionar que para la construcción del instrumento se partió de un doble proceso: a) de su justificación teórica a partir del modelo psicológico de salud biológica, y b) de la revisión de los reactivos por parte de jueces expertos, tanto en el manejo del dominio teórico y conceptual del modelo como en la atención de pacientes VIH positivos.

Para los participantes del CISAL la aplicación del instrumento se llevó a cabo en el auditorio del mismo centro, mientras que para los participantes del Hospital "Adolfo Félix Lostaunau" se llevó a cabo en un área designada para la atención de las personas VIH positivas. A todos los participantes se les explicó el motivo del estudio, solicitándoles su colaboración de manera voluntaria; se les aclaró que sus respuestas 
serían anónimas y confidenciales, y que los resultados serían utilizados con fines de investigación. Todas las personas ofrecieron su consentimiento para colaborar, aunque del total de 62 personas originales se eliminaron dos, en virtud de que en ese momento una no se encontraba bajo régimen de tratamiento antirretroviral y la otra, porque su historial médico estaba incompleto.

En el análisis de los resultados se empleó el paquete estadístico SPSS para Windows, versión 8.0. En primer lugar, se procedió a extraer las puntuaciones totales de respuesta para cada uno de los reactivos, con cuatro y cinco opciones de respuesta. Posteriormente, se obtuvieron los valores mínimos (primero de los cuartiles) y máximos (cuarto de los cuartiles), los cuales fueron recodificados, para finalmente analizar su grado de dificultad y discriminación, empleándose para dicho propósito la prueba $t$; en segundo lugar, se midió la validez de constructo a través de un análisis de su estructura factorial, con el método de componentes principales y rotación varimax (normalización con Kaiser), tomando en cuenta únicamente los reactivos que con la prueba $t$ habían demostrado ser significativos y obtuvieron pesos factoriales iguales a o mayores que 0.40; en tercer lugar, se evaluó la confiabilidad del instrumento recurriendo al estadístico alfa de Cronbach.

\section{Resultados}

Tal y como se muestra en el cuadro I los resultados obtenidos con la prueba $t$ demuestran que con excepción de dos reactivos (números 11 y 12), el resto discriminaron correctamente, puesto que en todos los casos se alcanzó significación estadística a $p \leq 0.01, p \leq 0.001$, $p<0.05$ y $p \leq 0.005$.

En cuanto a la validez de constructo del instrumento, del total de los 22 reactivos con los que se trabajó con este procedimiento, con el análisis factorial se eliminaron tres, el número 22, debido a que obtuvo un peso factorial menor que 0.40 , en tanto que los números 17 y 18 se agruparon en un índice (conductas relacionadas con bienestar físico y psicológico), se toma en cuenta que cada factor debe contener al menos tres reactivos. Tal y como se observa en el cuadro II, se conformaron tres factores, que respectivamente explican $22.96,12.62$ y $12.40 \%$ de la varianza, mientras que en conjunto explican $47.99 \%$ de la varianza. En los tres factores se alcanzaron valores del alfa de Cronbach de $0.879,0.750$ y 0.750 , respectivamente, en tanto que para el instrumento en su conjunto se obtuvo un alfa global de 0.856 , por lo que podemos concluir que el instrumento posee validez de constructo y es altamente confiable.
Cuadro I

ANÁLISIS DE DIFICULTAD Y DISCRIMINACIÓN DE LOS reactivos con base en la prueba t. México, 2002

\begin{tabular}{|c|c|c|c|c|c|}
\hline Reactivo & Media & $D E$ & $t$ & $\mathrm{GL}$ & Significación* \\
\hline 1 & 4.59 & 0.97 & -2.847 & 37 & $0.013^{*}$ \\
\hline 2 & 4.71 & 0.95 & -2.647 & 37 & $0.018^{*}$ \\
\hline 3 & 4.63 & 1.10 & -2.736 & 37 & $0.016^{*}$ \\
\hline 4 & 4.66 & 1.03 & -2.801 & 37 & $0.014^{*}$ \\
\hline 5 & 4.47 & 1.32 & -3.603 & 37 & $0.003^{* * * *}$ \\
\hline 6 & 3.15 & 1.10 & -3.141 & 37 & $0.007^{*}$ \\
\hline 7 & 4.59 & 0.98 & -5.746 & 29 & $0.000 * *$ \\
\hline 8 & 4.34 & 1.28 & -4.187 & 37 & $0.001 * *$ \\
\hline 9 & 4.20 & 1.37 & -3.282 & 37 & $0.005^{* * * *}$ \\
\hline 10 & 4.76 & 0.86 & -2.162 & 37 & $0.048 * * *$ \\
\hline 11 & 4.83 & 0.75 & -1.655 & 37 & 0.120 (ns) \\
\hline 12 & 3.73 & 0.67 & -1.948 & 29 & 0.072 (ns) \\
\hline 13 & 3.53 & 1.10 & -3.384 & 29 & $0.004 * * * *$ \\
\hline 14 & 3.39 & 1.11 & -7.341 & 29 & $0.000 * *$ \\
\hline 15 & 3.03 & 1.03 & -4.324 & 29 & $0.000 * *$ \\
\hline 16 & 3.64 & 0.87 & -2.709 & 29 & $0.017^{*}$ \\
\hline 17 & 2.80 & 1.26 & -2.878 & 29 & $0.008 *$ \\
\hline 18 & 3.24 & 1.12 & -4.561 & 29 & $0.000 * *$ \\
\hline 19 & 3.41 & 1.16 & -3.452 & 29 & $0.004^{* * * * *}$ \\
\hline 20 & 3.41 & 0.98 & -4.836 & 29 & $0.000 * *$ \\
\hline 21 & 4.31 & 1.37 & -4.116 & 37 & $0.001^{* *}$ \\
\hline 22 & 4.14 & 1.38 & -4.964 & 37 & $0.000 * *$ \\
\hline 23 & 4.29 & 1.31 & -2.941 & 37 & $0.011^{*}$ \\
\hline 24 & 4.36 & 1.24 & -2.738 & 37 & $0.016^{*}$ \\
\hline
\end{tabular}

$* p \leq .01 ; * * p \leq .001 ; * * * p<.05 ; * * * * p \leq .005$.

\section{Discusión}

Una vez cubiertos los principales requisitos que se contemplan en la elaboración de un instrumento, tanto en lo que se refiere a su sustento teórico como a su fundamentación psicométrica, ${ }^{13,14}$ de acuerdo con los resultados descritos previamente la encuesta para medir competencias conductuales en personas VIH positivas evidenció: a) claridad y buena discriminación de los reactivos; b) poseer validez de constructo, si bien es cierto que ésta arrojó una estructura factorial ligeramente diferente a la planteada originalmente, y c) un alfa de Cronbach que demuestra que el instrumento es altamente confiable.

No obstante estas conclusiones, es conveniente destacar dos limitaciones observadas en este estudio. En primer término, el número limitado de participantes que se encuentran bajo un régimen de tratamiento 


\section{Cuadro II \\ Análisis factorial exploratorio (PRUeba de VAlidez del Constructo competencias CONDUCtUales en PERSONAS VIH POSITIVAS) CON EL MÉTODO DE COMPONENTES PRINCIPALES Y ROTACIÓN VARIMAX (normalización con Kaiser) y valores del alfa de Cronbach. México, 2002}

Factor 1. Conocimientos sobre la enfermedad y el tratamiento

5. Sé cuáles son las principales infecciones oportunistas 0.846

4. Sé cuáles son las etapas por las que se pasa de VIH a SIDA

0.827

7. Conozco la función de los medicamentos antirretrovirales

0.818

2. Estoy informado de lo que significan los resultados de laboratorio

0.798

10. Sé qué medicamentos consumo y por qué debo tomarlos

0.765

3. Sé los cambios que puede sufrir mi cuerpo por el avance del SIDA

0.691

1. Hago lo necesario para conocer sobreVIH/SIDA

9. Sé que hay medicamentos que pueden tener efectos "cruzados"

0.681

8. Estoy informado sobre los efectos secundarios de los medicamentos

Valores propios $=6.41 ;$ Varianza explicada $=22.90 \% ; \mathrm{Alfa}=0.879$

Factor 2. Conductas de cuidado de salud y de relaciones sociales

23. Las relaciones sociales en mi trabajo no han cambiado 0.728

24. No tengo problemas para relacionarme con personas desconocidas

0.682

21. Las relaciones con mis familiares no han cambiado

0.651

16. Desde que vivo con VIH/SIDA evito el consumo alcohol o drogas

0.599

19. Desde que vivo con VIH/SIDA tengo relaciones sexuales protegidas

0.558

Valores propios $=3.51 ;$ Varianza explicada $=12.62 \% ; \mathrm{Alfa}=0.750$

Factor 3. Conductas relacionadas con la atención médica y seguimiento de instrucciones

14. Si los medicamentos me caen mal, solicito y acudo a la cita con mi médico

0.815

6. Si me siento mal solicito y acudo a la cita con el médico/psicólogo

20. Desde que vivo con VIH/SIDA pido al médico/psicólogo que me den tiempo

0.779

15. Desde que vivo con VIH/SIDA sigo una dieta apropiada

0.474

13. Tomo los medicamentos como se me indicó (con agua 0 alimentos)

0.423

Valores propios $=1.94$; Varianza explicada $=12.40 \% ; \mathrm{Alfa}=0.750$

Indice de conductas relacionadas con bienestar físico y psicológico

17. Desde que vivo con VIH/SIDA hago ejercicio físico dosificado

18. Desde que vivo con VIH/SIDA realizo actividades que me mantienen tranquila/o y relajada/o

Varianza glo bal explicada $=47.99 \%$

Alfa global estandarizada $=0.856$

con medicamentos antirretrovirales, si bien es cierto que habría que hacer la acotación de que se trata de poco más de $50 \%$ de las personas $\mathrm{VIH}$ positivas que actualmente se encuentran recibiendo atención en ambas instituciones; en segundo lugar, que en virtud de que se trata de un autorreporte de la conducta, habría que establecer en el futuro su utilidad y valor reales, por ejemplo probar su validez concurrente respecto de otras medidas de la conducta, casos de los registros observacionales, el conteo de píldoras, el autorregis- tro cotidiano de conductas relacionadas con el consumo de medicamentos, según las especificaciones del personal de salud, principalmente.

Teniendo en cuenta estas limitaciones y puesto que nuestro interés consistió inicialmente en probar las propiedades psicométricas del instrumento, consideramos pertinente que para futuras investigaciones se contemple una muestra más amplia de personas VIH positivas y cumplir con una tarea adicional: tratar de identificar posibles covariaciones entre esa $u$ otras 
medidas psicológicas (estilos interactivos, adhesión al tratamiento y apoyo social, por ejemplo) con medidas biológicas como el tiempo de infección, estadio clínico según los Centros para el Control de las Enfermedades (CDC, por sus siglas en inglés), carga viral, número de linfocitos CD4 y tipo de tratamiento que reciben los pacientes. Se trata, finalmente, a partir de los resultados que deriven de futuras investigaciones, de instrumentar programas de intervención médico y psicológica que correspondan a las necesidades de los pacientes en ambos rubros ${ }^{15} \mathrm{y}$ cuyos objetivos sean:

a) mejorar la práctica de conductas de adhesión al tratamiento; ;6-17

b) promover la práctica de conductas orientadas al cuidado y mantenimiento de la salud el mayor tiempo posible, ${ }^{18-19}$ así como de otras tendientes a eliminar estados emocionales negativos, y

c) mejorar la calidad de las relaciones sociales y la calidad de vida. ${ }^{20-22}$

\section{Referencias}

1. Remor EA.Valoración de la adhesión al tratamiento antirretroviral en pacientes VIH +. Psicothema 2000;14:262-267.

2. Remor EA, Carrobles JA.Versión española de la escala de estrés percibido (PSS-14): estudio psicométrico en una muestraVIH +. Ansiedad y Estrés 2001;7:195-201.

3. Kalichman SC, D iFonzo K, Austin J, LukeW, Rompa D. Prospective study of emotional reactions to change in HIV viral load.AIDS Patient Care \& Stds 2002;16:113-120.

4. Remor EA.A poyo social y calidad de vida en la infección por el VIH. Atención Primaria 2002;30:143-148.

5. O rtiz F,Arranz P, Remor EA. Presentación de un modelo de evaluación del grado de sufrimiento percibido en una muestra de pacientes hemofílicos VIH +. Revista Electrónica de Psicología 2000; 4:1-6.

6. Jenkins RA, Patterson TL. HIV locus of control and adaptation to seropositivity. Appli Soc Psychol 1998;2:95-98.
7. G onzález-Puente JA, Sánchez-Sosa JJ. Systematic assessment of quality of life in HIV-positive patients at three clinical stages of the infection. International Journal of C linical and Health Psychology 2001;1:519-528. 8. Ribes E, Sánchez S. El problema de las diferencias individuales: un análisis conceptual de la personalidad. En: Ribes E, Ed. Problemas conceptuales en el análisis del comportamiento humano. México, DF: Editorial Trillas, 1990.

9. Bayés R.Variaciones sobre un modelo psicológico de prevención de enfermedad y su aplicación al caso del SIDA.Acta Comportamentalia 1992;0:33-50.

10. Ribes E. Psicología y salud: un análisis conceptual. Barcelona: Martínez Roca, 1990.

11. Bayés R, Ribes $E$. Un modelo psicológico de prevención de enfermedad: su aplicación al caso del SIDA. En: Piña JA, Comp. Psicología y salud: aportes del análisis de la conducta. Hermosillo, México: Editorial UN ISO N, 1992.

12. Rodríguez ML, Moreno D, Robles S, Díaz-González E. El sida desde el modelo psicológico de salud biológica. Psicología y Salud 2000;10:161175.

13. Corral V. Condiciones que incrementan la precisión de los reportes verbales del comportamiento. Revista Latina de Pensamiento y Lenguaje 1999;7:107-124.

14. Cortada de Kohan N . Importancia de la investigación psicométrica. Revista Latinoamericana de Psicología 2002;34:229-240.

15. Piña JA, Togawa C, Coronado M, Fierros LE. Evaluación de un programa de intervención médico-psicológica dirigido a personas que viven con VIH/SIDA. Revista Chilena de Psicología 2000;21:119-129. 16. W agner GJ, Remien RH, C arballo-D iéguez A, D olezal C. Correlates of adherence to combination antiretroviral therapy among members of HIV-positive mixed status couples.AIDS C are 2002;14:105-109. 17. Bangsber DR, Bronstone A, Hofmann R.A computer-based assessment detects regimen misunderstandings and nonadherence for patients on HIV antiretroviral therapy.AIDS C are 2002;14:3-15.

18. Carrobles JA, Remor E, Rodríguez-Alzamora L. Afrontamiento, apoyo social percibido y distrés emocional en pacientes con infección por VIH. Psicothema 2003;15:420-426.

19. Remor E, Carrobles JA,Arranz P, Martínez-D onate A, U lla S. Ansiedad y percepción de control en la infección por VIH y SIDA. Psicología Conductual 2001;9:323-336.

20.Vosvick M, Gore-Felton C, Koopman C, Thoresen C, Krumboltz J, Spiegel D. Maladaptative coping strategies in relation to quality of life among HIV+ adults.AIDS and Behavior 2002;6:97-106.

21. Bayés R. Tras la $14^{\mathrm{a}}$ Conferencia Internacional sobre el SID A, ¿cuánto sufrimiento nos espera todavía? Revista Española de Drogodependencias 2002;27:226-235.

22. Bayés R. Infección por VIH : aspectos psicológicos. En: Bueno F, Nájera R, Eds. Salud pública y SIDA. Madrid: Ediciones D oyma; 2001. 